\title{
The Structured Singular Value for Linear Input/Output Operators
}

\author{
Hari Bercovici and Ciprian Foias \\ Department of Mathematics \\ Indiana University \\ Bloomington, Indiana 47405 \\ Allen Tannenbaum \\ Department of Electrical Engineering \\ University of Minnesota \\ Minneapolis, Minnesota 55455
}

\begin{abstract}
In this paper, we employ a lifting method introduced by the authors in order to study the structured singular value applied to input/output operators of control systems. We moreover give a new criterion which guarantees that the structured singular value equals its upper bound defined by $D$-scalings.
\end{abstract}

Key words. Structured singular value, time varying perturbations, lifting, robust control

AMS subject classifications. 93B35, 93C05

* This work was supported in part by grants from the Research Fund of Indiana University, by the National Science Foundation DMS-8811084, ECS-9122106, by the Air Force Office of Scientific Research AFOSR AF/F49620-94-1-00S8DEF, and by the Army Research Office DAAH04-94-G-0054 and DAAH04-93-G-0332. 


\section{Introduction}

Let $A$ be a linear operator on a Hilbert space $\mathcal{E}$, and let $\Delta$ be an algebra of operators on $\mathcal{E}$. The structured singular value of $A$ (relative to $\Delta$ ) is the number

$$
\mu_{\Delta}(A)=1 / \inf \{\|X\|: X \in \Delta,-1 \in \sigma(A X)\} .
$$

This quantity was introduced by Doyle and Safonov $[6,12]$ under a more restrictive context, and it has proved to be a powerful tool in robust system analysis and design. In system analysis, the structured singular value gives a measure of robust stability with respect to certain perturbation measures. Unfortunately, $\mu_{\Delta}(A)$ is very difficult to calculate, and in practice an upper bound for it is used. This upper bound is defined by

$$
\widehat{\mu}_{\Delta}(A):=\inf \left\{\left\|X A X^{-1}\right\|: X \in \Delta^{\prime}, X \text { invertible }\right\},
$$

where $\Delta^{\prime}$ is the commutant of the algebra $\Delta$.

In $[1,5]$, we formulated a lifting technique for the study of the structured singular value. The basic idea is that $\widehat{\mu}_{\Delta}(A)$ can be shown to be equal to the structured singular value of an operator on a bigger Hilbert space. (In [1] this was done for finite dimensional Hilbert spaces, and then in [5] this was extended to the infinite dimensional case.) The problem with these results is that the size of the ampliation necessary to get $\hat{\mu}_{\Delta}(A)$ equal to a structured singular value, was equal to the dimension of the underlying Hilbert space. Hence in the infinite dimensional case we needed an infinite ampliation. In this work, we will show that in fact, one can always get by with a finite lifting. (Note that in this paper we will be using the terms "ampliation" and "lifting" interchangeably.) For the block diagonal algebras of interest in robust control, the ampliation only depends on the number of blocks of the given perturbation structure. (See Theorem 1 below.) We moreover, give a new result when $\hat{\mu}_{\Delta}(A)=\mu_{\Delta}(A)$, that is, when no lifting is necessary and so $\hat{\mu}_{\Delta}(A)$ gives a nonconservative measure of robustness. (See Theorem 2.) This is then used to derive an elegant result of Shamma [13, 14] on Toeplitz operators. See also $[7,9,10]$ for related work in this area.

We now briefly sketch the contents of this paper. In Section 2, we give some background results which will be needed in the proof of Theorem 1 . In Section 3, we derive a number of useful facts about the relative numerical range. Then in Section 4, we state and prove our new version of the lifting 
theorem relating the structured singular value and its upper bound. In Section 5, we give new conditions when $\mu=\hat{\mu}$. These are applied in Section 6 , to give a new proof of the aforementioned result of Shamma. Finally, in Section 7, we give a system-theoretic interpretation of our lifting methodology.

\section{Preliminary Results}

Denote by $\mathcal{L}(\mathcal{E})$ the algebra of all bounded linear operators on the (complex, separable) Hilbert space $\mathcal{E}$. Fix an operator $A \in \mathcal{L}(\mathcal{E})$ and a subalgebra $\Delta \subset \mathcal{L}(\mathcal{E})$. The numbers $\mu_{\Delta}(A)$ and $\hat{\mu}_{\Delta}(A)$ have already been defined in the Introduction. Observe that $\Delta \subset \Delta^{\prime \prime}$ and $\Delta^{\prime \prime \prime}=\left(\Delta^{\prime \prime}\right)^{\prime}=\Delta^{\prime}$ so that we have the inequalities

$$
\mu_{\Delta}(A) \leq \mu_{\Delta^{\prime \prime}}(A), \quad \widehat{\mu}_{\Delta}(A)=\widehat{\mu}_{\Delta^{\prime \prime}}(A)
$$

Observe that the algebras $\Delta$ considered in [6] consisted of block diagonal matrices, so our approach is more general in this respect. In the following proposition we summarize some of the elementary properties of $\mu_{\Delta}$; see Doyle

[6] or [1] for proofs. We will denote by $\|T\|_{\text {sp }}$ the spectral radius of the operator $T$.

Lemma $1 \quad$ (i) $\mu_{\Delta}(A)=\sup \left\{\|A X\|_{\mathrm{sp}}: X \in \Delta,\|X\| \leq 1\right\}$;

(ii) $\mu_{\Delta}$ is upper semicontinuous;

(iii) If $\mathcal{E}$ is finite dimensional, then $\mu_{\Delta}$ is continuous;

(iv) $\mu_{\Delta}(A) \leq \widehat{\mu}_{\Delta}(A)$.

In our study we will need further singular values which we now define. For $n \in\{1,2, \ldots, \infty\}$ we denote by $\mathcal{E}^{(n)}$ the orthogonal sum of $n$ copies of $\mathcal{E}$, and by $T^{(n)}$ the orthogonal of $n$ copies of $T \in \mathcal{L}(\mathcal{E})$. Operators on $\mathcal{E}^{(n)}$ can be represented as $n \times n$ matrices of operators in $\mathcal{L}(\mathcal{E})$, and $T^{(n)}$ is represented by a diagonal matrix, with diagonal entries equal to $T$.

Denote by $\Delta_{n}$ the algebra of all operators on $\mathcal{E}^{(n)}$ whose matrix entries belong to $\Delta$, and observe that $\left(\Delta_{n}\right)^{\prime \prime}=\left(\Delta^{\prime \prime}\right)_{n}$, and $\left(\Delta_{n}\right)^{\prime}=\left(\Delta^{\prime}\right)^{(n)}=\left\{T^{(n)}\right.$ : $\left.T \in \Delta^{\prime}\right\}$. Therefore we will denote these algebras by $\Delta_{n}^{\prime \prime}$ and $\Delta_{n}^{\prime}$, respectively. 
Lemma 2 For every finite number $n$ we have

$$
\mu_{\Delta}(A) \leq \mu_{\Delta_{n}}\left(A^{(n)}\right) \leq \mu_{\Delta_{n+1}}\left(A^{(n+1)}\right) \leq \mu_{\Delta_{\infty}}\left(A^{(\infty)}\right) \leq \widehat{\mu}_{\Delta}(A)
$$

and

$$
\mu_{\Delta^{\prime \prime}}(A) \leq \mu_{\Delta_{n}^{\prime \prime}}\left(A^{(n)}\right) \leq \mu_{\Delta_{n+1}^{\prime \prime}}\left(A^{(n+1)}\right) \leq \mu_{\Delta_{\infty}^{\prime \prime}}\left(A^{(\infty)}\right) \leq \widehat{\mu}_{\Delta}(A)
$$

Proof. It clearly suffices to prove the first sequence of inequalities. Observe that for every $X \in \Delta_{n}$ and for $m>n$ we can define an operator $Y \in \Delta_{m}$ by $Y=X \oplus 0$. Clearly $\sigma\left(A^{(n)} X\right)=\sigma\left(A^{(m)} Y\right) \cup\{0\}$ and hence $-1 \in \sigma\left(A^{(n)} X\right)$ implies $-1 \in \sigma\left(A^{(m)} Y\right)$. Since $\mu_{\Delta}(A)=\mu_{\Delta_{1}}\left(A^{(1)}\right)$, this proves the first three inequalities. The last one follows because $\mu_{\Delta_{\infty}}\left(A^{(\infty)}\right) \leq \hat{\mu}_{\Delta_{\infty}}\left(A^{(\infty)}\right)=\hat{\mu}_{\Delta}(A)$. 2

We will now state (without proof) several results from $[1,2,3,4,5]$ which we will need in the sequel.

Lemma $3([5])$ Let $A$ be finite dimensional $\mathrm{C}^{*}$-algebra. Then $A$ has only finitely many equivalence classes of cyclic representations.

Lemma 4 ([5]) Let the sequence $Y_{j}$ of operators on $\mathcal{H}$, and the sequence $h_{j} \in \mathcal{H}$ satisfy

(i) $\sup _{j} \operatorname{rank} Y_{j}<\infty, \sup _{j}\left\|Y_{j}\right\|<\infty$;

(ii) $\lim _{j \rightarrow \infty}\left\|\left(Y_{j}-I\right) h_{j}\right\|=0$;

(iii) $\lim _{j \rightarrow \infty}\left\|h_{j}\right\|=1$.

Then $\lim \inf _{n \rightarrow \infty}\left\|Y_{j}\right\|_{\mathrm{sp}} \geq 1$.

Lemma 5 ([4]). Let $\mathcal{H}$ be a Hilbert space, $T \in \mathcal{L}(\mathcal{H})$, and $D_{j} \in \mathcal{L}(\mathcal{H})$ invertible so that

$$
T_{0}=\lim _{j \rightarrow \infty} D_{j} T D_{j}^{-1} .
$$

If the set $\left\{D_{j}, D_{j}^{-1}: j=1,2, \ldots\right\}$ is contained in a finite dimensional subspace, then $\left\|T_{0}\right\|_{\mathrm{sp}}=\|T\|_{\mathrm{sp}}$. 


\section{Relative Numerical Range}

We will also need some results in what follows about the relative numerical range. Let $\mathcal{H}$ be a complex separable Hilbert space, and let $\mathcal{L}(\mathcal{H})$ denote the set of bounded linear operators on $\mathcal{H}$. Let $T_{1}, \ldots, T_{m}, Q \in \mathcal{L}(\mathcal{H})$. Then we define the following relative numerical ranges:

$$
\begin{gathered}
W_{Q}\left(T_{1}, \ldots, T_{m}\right):=\left\{\lambda \in \mathbf{C}^{n}, \lambda=\lim _{n \rightarrow \infty}\left(\left\langle T_{j} h_{n}, h_{n}\right\rangle\right)_{j=1}^{m}:\right. \\
\left.h_{n} \in \mathcal{H},\left\|h_{n}\right\|=1, \lim _{n \rightarrow \infty}\left\|Q h_{n}\right\|=0\right\},
\end{gathered}
$$

and

$$
\begin{array}{r}
W_{Q}^{0}\left(T_{1}, \ldots, T_{m}\right):=\left\{\lambda \in \mathbf{C}^{n}, \lambda=\lim _{n \rightarrow \infty}\left(\left\langle T_{j} h_{n}, h_{n}\right\rangle\right)_{j=1}^{m}:\right. \\
\left.h_{n} \in \mathcal{H},\left\|h_{n}\right\|=1, \lim _{n \rightarrow \infty}\left\|Q h_{n}\right\|=0, h_{n} \rightarrow 0 \text { weakly }\right\} .
\end{array}
$$

Lemma $6 W_{Q}^{0}\left(T_{1}, \ldots, T_{m}\right)$ is a compact convex subset of $\mathbf{C}^{m}$.

Proof. The compactness is immediate since $W_{Q}^{0}\left(T_{1}, \ldots, T_{m}\right)$ is a closed bounded subset of $\mathbf{C}^{m}$. As for the convexity, let $\lambda=\left(\lambda_{1}, \ldots, \lambda_{m}\right), \mu=$ $\left(\mu_{1}, \ldots, \mu_{m}\right) \in W_{Q}^{0}\left(T_{1}, \ldots, T_{m}\right)$, and let the sequences of unit vectors

$$
\left\{h_{n}\right\}_{n=1}^{\infty},\left\{k_{n}\right\}_{n=1}^{\infty} \subset \mathcal{H}
$$

satisfy

$$
\begin{gathered}
\lambda_{j}=\lim _{n \rightarrow \infty}\left\langle T_{j} h_{n}, h_{n}\right\rangle, \\
\mu_{j}=\lim _{n \rightarrow \infty}\left\langle T_{j} k_{n}, k_{n}\right\rangle, j=1, \ldots, m, \\
\lim _{n \rightarrow \infty}\left\|Q h_{n}\right\|=0=\lim _{n \rightarrow \infty}\left\|Q k_{n}\right\|, h_{n} \rightarrow 0, k_{n} \rightarrow 0 \text { weakly. }
\end{gathered}
$$

Next for $n$ fixed choose $N_{n} \geq n$ such that

$$
\left|\left\langle h_{n}, k_{N_{n}}\right\rangle\right| \leq \frac{1}{n},\left|\left\langle T_{j} h_{n}, k_{N_{n}}\right\rangle\right|+\left|\left\langle T_{j}^{*} h_{n}, k_{N_{n}}\right\rangle\right| \leq \frac{1}{n}, j=1,2, \ldots, m .
$$

Then for any $\theta \in[0,1]$

$$
g_{n}:=\sqrt{\theta} h_{n}+\sqrt{1-\theta} k_{N_{n}}, n \geq 2,
$$


satisfies the following conditions:

$$
\begin{gathered}
\left\|g_{n}\right\|^{2}=1+2 \sqrt{\theta(1-\theta)} \Re\left\langle h_{n}, k_{N_{n}}\right\rangle \rightarrow 1,\left\|g_{n}\right\|^{2} \geq \frac{1}{2}, \\
g_{n} \rightarrow 0 \text { weakly, }\left\|Q g_{n}\right\| \rightarrow 0, \\
\left|\left\langle T_{j} g_{n}, g_{n}\right\rangle-\theta \lambda_{j}-(1-\theta) \mu_{j}\right| \leq \theta\left|\left\langle T_{j} h_{n}, h_{n}\right\rangle-\lambda_{j}\right|+(1-\theta)\left|\left\langle T_{j} k_{N_{n}}, k_{N_{n}}\right\rangle-\mu_{j}\right|+ \\
\sqrt{\theta(1-\theta)} \frac{1}{n} \rightarrow 0 \text { as } n \rightarrow \infty .
\end{gathered}
$$

Thus replacing the $g_{n}$ by $g_{n} /\left\|g_{n}\right\|$ we immediately conclude that

$$
\theta \lambda+(1-\theta) \mu \in W_{Q}^{0}\left(T_{1}, \ldots, T_{m}\right),
$$

as required. 2

Lemma $7 W_{Q}\left(T_{1}, \ldots, T_{m}\right)$ is the union of all segments

$$
\{\theta \lambda+(1-\theta) \mu: 0 \leq \theta \leq 1\}
$$

where $\lambda \in W_{Q}^{0}\left(T_{1}, \ldots, T_{m}\right)$ and $\mu=\left(\left\langle T_{j} h, h\right\rangle\right)_{j=1}^{m}$ for some $h \in \operatorname{ker} Q,\|h\|=$ 1.

Proof. Let $\theta, \lambda=\left(\lambda_{j}\right)_{j=1}^{m}, \mu=\left(\mu_{j}\right)_{j=1}^{m}$ be as above and let the sequence $\left\{h_{n}\right\}_{n=1}^{\infty} \subset \mathcal{H}$ satisfy $\left\|h_{n}\right\|=1, h_{n} \rightarrow 0$ weakly, $Q h_{n} \rightarrow 0$ strongly, and $\left\langle T_{j} h_{n}, h_{n}\right\rangle \rightarrow \lambda_{j}$ for $j=1, \ldots, m$. Then as in the proof of Lemma 6 , we obtain that

$$
g_{n}=\sqrt{\theta} h_{n}+\sqrt{1-\theta} h, n=1,2, \ldots
$$

satisfies the conditions

$$
\left\|g_{n}\right\| \rightarrow 1, \quad\left\|Q g_{n}\right\| \rightarrow 0
$$

and

$$
\left\langle T_{j} g_{n}, g_{n}\right\rangle \rightarrow \theta \lambda_{j}+(1-\theta) \mu_{j}, \quad j=1, \ldots, m
$$

Therefore

$$
\theta \lambda+(1-\theta) \mu \in W_{Q}\left(T_{1}, \ldots, T_{m}\right)
$$


Conversely, if $\psi=\left(\psi_{j}\right)_{j=1}^{m} \in W_{Q}\left(T_{1}, \ldots, T_{m}\right)$, then

$$
\psi_{j}=\lim _{n \rightarrow \infty}\left\langle T_{j} g_{n}, g_{n}\right\rangle, j=1, \ldots, m,
$$

for some sequence $\left\{g_{n}\right\}_{n=1}^{\infty} \subset \mathcal{H}$ such that $\left\|g_{n}\right\|=1,\left\|Q g_{n}\right\| \rightarrow 0$. Without loss of generality we can assume that $g_{n}$ converges weakly to some $h^{\prime} \in \operatorname{ker} Q$.

If $\left\|h^{\prime}\right\|=1$ then $g_{n}$ converges strongly to $h^{\prime}$ and $\psi_{j}=\left\langle T_{j} h^{\prime}, h^{\prime}\right\rangle, j=$ $1,2, \ldots, m$. Clearly then

$$
\psi=\theta \lambda+(1-\theta)\left(\left\langle T_{j} h, h\right\rangle\right)_{j=1}^{m}
$$

with $h=h^{\prime}, \theta=0$, and $\lambda \in W_{Q}^{0}\left(T_{1}, \ldots, T_{m}\right)$. If $\left\|h^{\prime}\right\|=0$ then $\psi$ belongs to $W_{Q}^{0}\left(T_{1}, \ldots, T_{m}\right)$ and hence $\psi=\theta \psi+(1-\theta) \mu$ with $\theta=1$ and $\mu$ arbitrary. Finally we consider the case when $h^{\prime} \neq 0$ and $\left\|h^{\prime}\right\| \neq 1$. The vectors $h_{n}=$ $\left(g_{n}-h^{\prime}\right) /\left\|g_{n}-h^{\prime}\right\|$ converge weakly to zero, $\left\|Q h_{n}\right\| \rightarrow 0$, and $h=h^{\prime} /\left\|h^{\prime}\right\|$ is a unit vector in $\operatorname{ker} Q$. Clearly then

$$
\psi_{j}=\left(1-\|h\|^{2}\right) \lim _{n \rightarrow \infty}\left\langle T_{j} h_{n}, h_{n}\right\rangle+\|h\|^{2}\left\langle T_{j} h, h\right\rangle, j=1, \ldots, m,
$$

and therefore

$$
\psi \in\left\{\theta \lambda+(1-\theta)\left(\left\langle T_{j} h, h\right\rangle\right)_{j=1}^{m}: 0 \leq \theta \leq 1\right\}
$$

where

$$
\lambda:=\lim _{n \rightarrow \infty}\left(\left\langle T_{j} h_{n}, h_{n}\right\rangle\right)_{j=1}^{m} \in W_{Q}^{0}\left(T_{1}, \ldots, T_{m}\right) .
$$

This concludes the proof. 2

Corollary 1 For all $T, Q \in \mathcal{L}(\mathcal{H})$, the set

$$
W_{Q}(T)=\left\{\lambda=\lim _{n \rightarrow \infty}\left\langle T h_{n}, h_{n}\right\rangle: h_{n} \in H,\left\|h_{n}\right\|=1, \lim _{n \rightarrow \infty}\left\|Q h_{n}\right\|=0\right\}
$$

is a compact convex set.

Proof. First notice that by an application of the classical Toeplitz-Hausdorff theorem to $T_{Q}:=P T \mid \operatorname{ker} Q$ where $P$ denotes orthogonal projection of $\mathcal{H}$ onto $\operatorname{ker} Q$, we see that the set

$$
W\left(T_{Q}\right)=\{\langle T h, h\rangle:\|h\|=1, h \in \operatorname{ker} Q\}
$$


is compact and convex. Therefore the convex hull of

$$
W_{Q}^{0}(T) \cup W\left(T_{Q}\right)
$$

is the union of all segments

$$
\{\theta \lambda+(1-\theta) \mu: 0 \leq \theta \leq 1\}
$$

where $\lambda$ and $\mu$ run over $W_{Q}^{0}(T)$ and $W\left(T_{Q}\right)$, respectively. But according to Lemma 7 , this union is precisely $W_{Q}(T) .2$

Remark. Corollary 1 was proven in [3] using a completely different argument, based on an approximation lemma which is of independent interest.

Finally, for the proof of our lifting theorem (to be given in Section 4), we will need the following elementary fact:

Lemma 8 Let $\mathcal{Z}$ denote a finite dimensional normed space, and let $S$ be a set of linear functionals on $\mathcal{Z}$. Suppose that for every $z \in \mathcal{Z}$ there exists a sequence $\ell_{n} \in S$ such that $\lim _{n \rightarrow \infty} \ell_{n}(z)=0$. Then there exists a sequence $\ell_{n}$ in the convex hull of $S$ such that $\lim _{n \rightarrow \infty}\left\|\ell_{n}\right\|=0$.

Proof. Since $\mathcal{Z}$ is finite dimensional, $S$ is contained in the dual $\mathcal{Z}^{\prime}$ of $\mathcal{Z}$. We may also assume that $S$ is a convex set. To prove the lemma we must show that the closure of $S$ contains zero. If it did not then the Hahn-Banach theorem would imply the existence of a vector $z \in \mathcal{Z}$ and of a number $\varepsilon>0$ such that $\Re \ell(z)>\varepsilon$ for all $\ell \in S$. This is contrary to the assumption of the lemma. 2

\section{Ampliations of Perturbations}

In this section, we will formulate and prove a new lifting result relating $\mu_{\Delta}(A)$ and $\hat{\mu}_{\Delta}(A)$. For finite dimensional $\mathcal{E}$, a lifting result of this type was first proven in [1]. The result was then generalized to the infinite dimensional case in [5]. (For another proof of this type of lifting result in finite dimensions, see [7].) In these theorems, the lifting or ampliation of the operator $A$ and perturbation structure $\Delta$ depends on the dimension of $\mathcal{E}$. Thus if $\mathcal{E}$ is infinite 
dimensional, we get an infinite lifting. In the new result proven below, we only have to lift up to the dimension of $\Delta^{\prime}$ which in the cases of interest in the control applications of this theory only depends on the number of blocks of the given perturbation structure.

The notation will be that used in Section 2.

Theorem 1 Assume that $\Delta^{\prime}$ is a *-algebra of finite dimension $n$. Then

$$
\widehat{\mu}_{\Delta}(A)=\mu_{\Delta_{n}^{\prime \prime}}\left(A^{(n)}\right)
$$

for every $A \in \mathcal{L}(\mathcal{E})$.

Proof. The argument starts as in the proof of Theorem 3 in [1], and of Theorem 1 of [5]. Without loss of generality, we may assume that $\hat{\mu}_{\Delta}(A)=1$. We must show that $\mu_{\Delta_{n}^{\prime \prime}}\left(A^{(n)}\right) \geq 1$. Choose a sequence of invertible operators $X_{j} \in \Delta^{\prime}$ such that $\left\|X_{j} A X_{j}^{-1}\right\| \rightarrow \widehat{\mu}_{\Delta}(A)$. Since $X_{j} A X_{j}^{-1}$ belongs to the finite dimensional space generated by $\Delta^{\prime} A \Delta^{\prime}$, we may assume that the sequence $X_{j} A X_{j}^{-1}$ converges to some operator $A_{0}$ such that $\left\|A_{0}\right\|=1$. Obviously $\left\|X A_{0} X^{-1}\right\| \geq\left\|A_{0}\right\|$ for every invertible operator $X \in \Delta^{\prime}$. In particular we have

$$
\left\|(I-X) A_{0}\left(I+X+X^{2}+\cdots\right)\right\| \geq 1
$$

for $X \in \Delta^{\prime}$ with $\|X\|<1$. Fix an operator $X \in \Delta^{\prime}$ and a sequence $\varepsilon_{j}>0$ converging to zero. There exist vectors $h_{j} \in \mathcal{E}$ with $\left\|h_{j}\right\|=1$, such that

$$
\left\|\left(I-\varepsilon_{j} X\right) A_{0}\left(I+\varepsilon_{j} X+\varepsilon_{j}^{2} X^{2}+\cdots\right) h_{j}\right\|^{2} \geq 1-\varepsilon_{j}^{2} .
$$

This can be rewritten as,

$$
\left\langle A_{0}^{*} A_{0} h_{j}, h_{j}\right\rangle+2 \varepsilon_{j} \Re\left\langle A_{0}^{*}\left(A_{0} X-X A_{0}\right) h_{j}, h_{j}\right\rangle+O\left(\varepsilon_{j}^{2}\right) \geq 1-\varepsilon_{j}^{2}
$$

or equivalently,

$$
2 \varepsilon_{j} \Re\left\langle A_{0}^{*}\left(A_{0} X-X A_{0}\right) h_{j}, h_{j}\right\rangle+O\left(\varepsilon_{j}^{2}\right) \geq\left\langle\left(I-A_{0}^{*} A_{0}\right) h_{j}, h_{j}\right\rangle-\varepsilon_{j}^{2} \geq-\varepsilon_{j}^{2} .
$$

Dividing by $\varepsilon_{j}$ and letting $\varepsilon_{j} \rightarrow 0$ as $j \rightarrow \infty$, we see from the last equation that

$$
\begin{aligned}
\left\langle\left(I-A_{0}^{*} A_{0}\right) h_{j}, h_{j}\right\rangle & \rightarrow 0, \\
\liminf _{j \rightarrow \infty} \Re\left\langle A_{0}^{*}\left(A_{0} X-X A_{0}\right) h_{j}, h_{j}\right\rangle & \geq 0 .
\end{aligned}
$$


We easily conclude that

$$
\liminf _{j \rightarrow \infty} \Re\left\langle\left(X-A_{0}^{*} X A_{0}\right) h_{j}, h_{j}\right\rangle \geq 0
$$

Set

$$
Q=I-A_{0}^{*} A_{0}, T=X-A_{0}^{*} X A_{0} .
$$

Then from $(1,3)$, we see that

$$
Q h_{j} \rightarrow 0, \quad \liminf _{j \rightarrow \infty} \Re\left\langle T h_{j}, h_{j}\right\rangle \geq 0 .
$$

Applying the above argument to $\zeta X$ for any $\zeta \in \partial \mathbf{D}$ (the unit circle), we see that there exists a sequence $h_{j}^{(\zeta)},\left\|h_{j}^{(\zeta)}\right\|=1$ such that

$$
Q h_{j}^{(\zeta)} \rightarrow 0, \liminf _{j \rightarrow \infty} \Re \zeta\left\langle T h_{j}, h_{j}\right\rangle \geq 0
$$

We claim that $0 \in W_{Q, 0}(T)$. Indeed, if this were not the case, Corollary 1 would imply the existence of $\zeta \in \partial \mathbf{D}$ such that

$$
\liminf _{j \rightarrow \infty} \Re \zeta\left\langle T h_{j}, h_{j}\right\rangle<0
$$

for all sequences of unit vectors $h_{j}$ such that $Q h_{j} \rightarrow 0$ contradicting (5).

Thus, we have shown that for each $X \in \Delta^{\prime}$, there exists a sequence of unit vectors $h_{j} \in \mathcal{E}$ such that

$$
\left(I-A_{0}^{*} A_{0}\right) h_{j} \rightarrow 0, \text { and }\left\langle\left(X-A_{0}^{*} X A_{0}\right) h_{j}, h_{j}\right\rangle \rightarrow 0 .
$$

Let

$$
\Delta_{\mathrm{sa}}^{\prime}:=\left\{X=X^{*}: X \in \Delta^{\prime}\right\} .
$$

Consider now a subspace $D \subset \Delta_{\mathrm{sa}}^{\prime}$ of real dimension $n-1$ such that $\Delta_{\mathrm{sa}}^{\prime}=$ $D+\mathbf{R} I$. Set $\mathcal{Z}=\left\{X-A_{0}^{*} X A_{0}: X \in D\right\}$, and for every unit vector $h \in \mathcal{E}$ define a linear functional $\ell(h)$ on $\mathcal{Z}$ by $\ell(h)(T)=\langle T h, h\rangle, T \in \mathcal{Z}$. Then Lemma 8 applied to the set $S_{k}=\left\{\ell(h):\left\|\left(I-A_{0}^{*} A_{0}\right) h\right\| \leq 1 / k\right\}$ implies the existence of linear functionals $\ell_{k}$ in the convex hull of $S_{k}$ such that $\left\|\ell_{k}\right\| \leq 1 / k$. Observe furthermore that the real dimension of $\mathcal{Z}$ is at most $n-1$. Then from a standard result (see e.g., [11], page 73), each $\ell_{k}$ is a 
convex combination of at most $n$ functionals $\ell(h)$, say $\ell_{k}=\sum_{j=1}^{n} \alpha_{j}^{(k)} \ell\left(h_{j}^{(k)}\right)$, where $\alpha_{j}^{(k)} \geq 0, \sum_{j=1}^{n} \alpha_{j}^{(k)}=1$, and the $h_{j}^{(k)}$ are unit vectors in $\mathcal{E}$, such that

$$
\left\|\left(I-A_{0}^{*} A_{0}\right) h_{j}^{(k)}\right\| \leq 1 / k .
$$

Let us define unit vectors vectors $u_{k} \in \mathcal{E}^{(n)}$ by

$$
u_{k}=\oplus_{j=1}^{n}\left(\alpha_{j}^{(k)}\right)^{1 / 2} h_{j}^{(k)},
$$

and observe that $\lim _{k \rightarrow \infty}\left\langle\left(X^{(n)}-A_{0}^{*(n)} X^{(n)} A_{0}^{(n)}\right) u_{k}, u_{k}\right\rangle=0$ for every $X \in \Delta^{\prime}$. Taking $X=Y^{*} Y$ we obtain

$$
\lim _{k \rightarrow \infty}\left(\left\|Y^{(n)} A_{0}^{(n)} u_{k}\right\|-\left\|Y^{(n)} u_{k}\right\|\right)=0
$$

for every $Y \in \Delta^{\prime}$.

Consider now the spaces $\mathcal{H}_{k}=\Delta_{n}^{\prime} A_{0}^{(n)} u_{k}$ and $\mathcal{K}_{k}=\Delta_{n}^{\prime} u_{k}$. Lemma $3 \mathrm{im}-$ plies that, by passing to appropriate subsequences, we may assume that all the representations $X \rightarrow X^{(n)} \mid \mathcal{H}_{k}$ (resp. $X \rightarrow X^{(n)} \mid \mathcal{K}_{k}$ ) are unitarily equivalent. It follows that we can find partial isometries $U_{k}, V_{k}$ in $\Delta_{n}^{\prime \prime}$ such that $U_{k} \mathcal{H}_{k}=\mathcal{H}_{1}$ and $V_{k} \mathcal{K}_{k}=\mathcal{K}_{1}$. Dropping again to appropriate subsequences, we may assume that the $\operatorname{limits} u=\lim _{k \rightarrow \infty} U_{k} A_{0}^{(n)} u_{k}$ and $v=\lim _{k \rightarrow \infty} V_{k} u_{k}$ exist. Then (8) implies that

$$
\left\|Y^{(n)} u\right\|=\left\|Y^{(n)} v\right\|
$$

for every $Y \in \Delta^{\prime}$. Therefore there exists a partial isometry $W \in \Delta_{n}^{\prime \prime}$ such that

$$
W Y^{(n)} u=Y^{(n)} v
$$

for every $Y \in \Delta^{\prime}$. Of course, $W$ can be chosen equal to zero on the orthogonal complement of $\Delta_{n}^{\prime} u$ and thus to have finite rank at most $n$. The partial isometries $R_{k}:=V_{k}^{*} W U_{k}$ are in $\Delta_{n}^{\prime \prime}$, they have uniformly bounded rank, and

$$
\lim _{k \rightarrow \infty}\left(R_{k} A_{0}^{(n)}-I\right) u_{k}=\lim _{k \rightarrow \infty} V_{k}^{*}\left(W U_{k} A_{0}^{(n)} u_{k}-V_{k} u_{k}\right)=0 .
$$

Therefore Lemma 4 implies that

$$
\liminf _{k \rightarrow \infty}\left\|R_{k} A_{0}^{(n)}\right\|_{\mathrm{sp}} \geq 1
$$


Finally, since $R_{k}$ commutes with $X^{(n)}, X \in \Delta^{\prime}$, and we have

$$
X_{j}^{(n)} R_{k} A^{(n)} X_{j}^{(n)-1} \rightarrow R_{k} A_{0}^{(n)}
$$

in norm as $j \rightarrow \infty$. Lemma 5 shows that

$$
\left\|R_{k} A_{0}^{(n)}\right\|_{\mathrm{sp}}=\left\|R_{k} A^{(n)}\right\|_{\mathrm{sp}} .
$$

Consequently, we have

$$
\liminf _{k \rightarrow \infty}\left\|R_{k} A^{(n)}\right\|_{\mathrm{sp}}=\liminf _{k \rightarrow \infty}\left\|R_{k} A_{0}^{(n)}\right\| \geq 1 .
$$

Thus,

$$
\mu_{\Delta_{n}^{\prime \prime}}\left(A^{(n)}\right) \geq \liminf _{k \rightarrow \infty}\left\|A^{(n)} X_{k}\right\|_{\mathrm{sp}} \geq 1=\widehat{\mu}_{\Delta}(A),
$$

which completes the proof of the theorem. 2

Remark. In the cases of interest in control,

$$
\Delta^{\prime \prime}=\Delta,
$$

and so one has from Theorem 1 that

$$
\mu_{\Delta_{n}}(A)=\hat{\mu}_{\Delta}(A) .
$$

\section{Conditions for $\mu=\hat{\mu}$}

In this section, we will discuss some new conditions when $\mu=\hat{\mu}$ without any need for lifting or ampliation. In the finite dimensional case, there have been some results of this kind, the most famous of which is that of Doyle [6], who showed that no lifting is necessary for perturbation structures with three or fewer blocks.

We begin by noting that in the proof of Theorem 1, we established a useful property of the critical operators $A_{0}$ in the closed $\Delta^{\prime}$ similarity orbit

$$
\overline{\mathcal{O}_{\Delta^{\prime}}(A)}=\overline{\left\{X A X^{-1}: X \in \Delta^{\prime}\right\}}
$$

of $A$. Namely, if we call critical any $A_{0} \in \overline{\mathcal{O}_{\Delta^{\prime}}(A)}$ satisfying

$$
\limsup _{\epsilon \downarrow 0}\left\|(I-\epsilon X) A_{0}(I-\epsilon X)^{-1}\right\| \geq\left\|A_{0}\right\|, \forall X \in \Delta^{\prime},
$$

then the first part of the proof of Theorem 1 establishes the following: 
Lemma 9 If $A_{0}$ is a critical operator in $\overline{\mathcal{O}_{\Delta^{\prime}}(A)}$, then it enjoys the following property $(\mathcal{O})$ :

$$
0 \in W_{Q}\left(\left\|A_{0}\right\|^{2} X-A_{0}^{*} X A_{0}\right), X \in \Delta^{\prime},
$$

where $Q=\left\|A_{0}\right\|^{2} I-A_{0}^{*} A_{0}$.

Indeed, property $(\mathcal{O})$ is a reformulation of equation $(6)$ in the case in which the norm of $A_{0}$ may be different from 1 .

The next lemma is the key step in adapting the proof of Theorem 1 in order to show that

$$
\mu_{\Delta}(A)=\widehat{\mu}_{\Delta}(A)
$$

in several interesting cases.

Lemma 10 Let $A_{0}$ be an operator on $\mathcal{E}$ which satisfies the essential version of property $(\mathcal{O})$, property $\left(\mathcal{O}^{0}\right)$, namely

$$
0 \in W_{Q}^{0}\left(\left\|A_{0}\right\|^{2} X-A_{0}^{*} X A_{0}\right), X \in \Delta^{\prime},
$$

where $Q=\left\|A_{0}\right\|^{2} I-A_{0}^{*} A_{0}$. Then there exists a sequence $\left\{h_{k}\right\}_{k=1}^{\infty} \subset \mathcal{E},\left\|h_{k}\right\|=$ $1, k=1,2, \ldots$, such that

$$
Q h_{k} \rightarrow 0 \text { strongly and }\left\langle\left(\left\|A_{0}\right\|^{2} X-A_{0}^{*} X A_{0}\right) h_{k}, h_{k}\right\rangle \rightarrow 0,
$$

for all $X \in \Delta^{\prime}$.

Proof. Without loss of generality we can assume that $\left\|A_{0}\right\|=1$. Let $X_{1}, \ldots, X_{n}$ be an algebraic basis of $\Delta^{\prime}$. (Note that $\Delta^{\prime}$ is finite dimensional.) Set $T_{j}:=X_{j}-A_{0}^{*} X_{j} A_{0}, j=1, \ldots, n$. Then by virtue of Lemma 6 , $W_{Q}^{0}\left(T_{1}, \ldots, T_{n}\right)$ is convex and compact. If $0 \notin W_{Q}^{0}\left(T_{1}, \ldots, T_{n}\right)$, there exists $\psi=\left(\psi_{1}, \ldots, \psi_{n}\right) \in \mathbf{C}^{n}$ and $\epsilon>0$ such that

$$
\Re \sum_{j=1}^{n} \psi_{j} \lambda_{j} \geq \epsilon, \quad \forall \lambda=\left(\lambda_{1}, \ldots, \lambda_{n}\right) \in W_{Q}^{0}\left(T_{1}, \ldots, T_{n}\right) .
$$

Set

$$
T=\sum_{j=1}^{n} \psi_{j} T_{j}
$$


Property $\left(\mathcal{O}^{0}\right)$ implies that there exists a sequence $\left\{g_{k}\right\}_{k=1}^{\infty} \subset \mathcal{E},\left\|g_{k}\right\|=1$, $g_{k} \rightarrow 0$ weakly such that $\left\langle T g_{k}, g_{k}\right\rangle \rightarrow 0$. Without loss of generality (by passing to a subsequence if necessary), we can assume that

$$
\left\langle T_{j} g_{k}, g_{k}\right\rangle \rightarrow \lambda_{j}, \quad(j=1, \ldots, n),
$$

for $k \rightarrow \infty$. Thus

$$
\lambda=\left(\lambda_{1}, \ldots, \lambda_{n}\right) \in W_{Q}^{0}\left(T_{1}, \ldots, T_{n}\right)
$$

Hence

$$
0 \leftarrow \Re\left\langle T g_{k}, g_{k}\right\rangle=\Re \sum_{j=1}^{n} \psi_{j}\left\langle T_{j} g_{k}, g_{k}\right\rangle \rightarrow \Re \sum_{j=1}^{n} \psi_{j} \lambda_{j} \geq \epsilon
$$

a contradiction. We therefore conclude that $0 \in W_{Q}^{0}\left(T_{1}, \ldots, T_{n}\right)$, i.e., there exists a sequence $\left\{h_{k}\right\}_{k=1}^{\infty} \subset \mathcal{E}$ satisfying the properties $\left\|h_{k}\right\|=1, k=1,2, \ldots$, $\left\|Q h_{k}\right\| \rightarrow 0, h_{k} \rightarrow 0$ weakly, and

$$
\left\langle\left(X_{j}-A_{0}^{*} X_{j} A_{0}\right) h_{k}, h_{k}\right\rangle=\left\langle T_{j} h_{k}, h_{k}\right\rangle \rightarrow 0
$$

for all $j=1,2, \ldots, n$. This implies that

$$
\left\langle\left(X-A_{0}^{*} X A_{0}\right) h_{k}, h_{k}\right\rangle \rightarrow 0,
$$

for all $X \in \Delta^{\prime} .2$

We can now state the second main result of this paper:

Theorem 2 If there exists a critical operator $A_{0}$ satisfying property $\mathcal{O}^{0}$ in the closed $\Delta^{\prime}$-orbit of $A$, then

$$
\mu_{\Delta^{\prime \prime}}(A)=\hat{\mu}_{\Delta}(A) .
$$

Proof. We only have to note that because of Lemma 10, in the proof of Theorem 1, we need not take direct sums. More precisely, referring to equation (7) in the proof of Theorem 1 , we can take $u_{k}=h_{k}$, where

$$
\left\{h_{k}\right\}_{k=1}^{\infty}
$$

is the sequence provided by Lemma 10 . The proof then proceeds exactly as in Theorem 1 with $A_{0}$ replacing $A_{0}^{(n)}, X$ replacing $X^{(n)}$, and $Y$ replacing $Y^{(n)} \cdot 2$ 
Remark. Under the hypotheses of Theorem 2, when $\Delta^{\prime \prime}=\Delta$ (which happens in all cases of interest in control), we have that

$$
\mu_{\Delta}(A)=\hat{\mu}_{\Delta}(A) .
$$

Let $L\left(\Delta^{\prime} A \Delta^{\prime}\right)$ denote the linear space generated by

$$
\Delta^{\prime} A \Delta^{\prime}=\left\{X A Y: X, Y \in \Delta^{\prime}\right\} .
$$

Obviously $L\left(\Delta^{\prime} A \Delta^{\prime}\right)$ is finite dimensional, and therefore closed. Hence $\overline{\mathcal{O}_{\Delta^{\prime}}(A)} \subset$ $L\left(\Delta^{\prime} A \Delta^{\prime}\right)$.

Corollary 2 If for every $B \in L\left(\Delta^{\prime} A \Delta^{\prime}\right), B \neq 0$, the norm of $B$ is not attained (that is, there is no $h \in \mathcal{H}$ such that $\|B h\|=\|B\|\|h\| \neq 0$ ), then

$$
\mu_{\Delta^{\prime \prime}}(A)=\hat{\mu}_{\Delta}(A) .
$$

Proof. The critical operator $A_{0}$ constructed in the first part of the proof of Theorem 1 belongs to $L\left(\Delta^{\prime} A \Delta^{\prime}\right)$, and therefore its norm is not attained. However in equation (6), we can assume that the sequence $\left\{h_{j}\right\}_{j=1}^{\infty}$ is weakly convergent, say $h_{j} \rightarrow h$ weakly. Without loss of generality, we may assume that $\left\|A_{0}\right\|=1$. Then (6) shows that

$$
\left(I-A_{0}^{*} A_{0}\right) h=0 .
$$

Therefore if $h \neq 0$, we would have

$$
\left\|A_{0} h\right\|^{2}=\|h\|^{2}=\left\|A_{0}\right\|^{2}\|h\|^{2} \neq 0,
$$

and so the norm of $A_{0}$ would be attained. We conclude that $h_{j} \rightarrow 0$ weakly, and so $A_{0}$ satisfies property $\left(\mathcal{O}^{0}\right)$. The required result now follows by Theorem 2. 2

Remark. Note that Corollary 2 applies only to infinite dimensional Hilbert spaces $\mathcal{E}$.

\section{Example.}


We would like to give an explicit example to which Corollary 2 applies. Let $A_{j}$ be an operator on a Hilbert space $\mathcal{E}_{j}(j=1, \ldots, n)$ for which the norm is not attained. (For example, take $\mathcal{E}_{j}=L^{2}((0,1))$ and $A_{j}$ the muliplication operator $f(x) \mapsto x f(x)$ for $x \in(0,1)$ and $f \in L^{2}((0,1))$.) Set

$$
\mathcal{E}:=\mathcal{E}_{1} \oplus \cdots \oplus \mathcal{E}_{n}
$$

and $\Delta$ the algebra of operators on $\mathcal{E}$ of the form

$$
\left[\begin{array}{cccc}
X_{1} & 0 & \ldots & 0 \\
0 & X_{2} & \ldots & 0 \\
\vdots & \vdots & \ddots & \vdots \\
0 & 0 & \ldots & X_{n}
\end{array}\right]
$$

with $X_{j} \in \mathcal{L}\left(\mathcal{E}_{j}\right), j=1,2, \ldots, n$. Then $\Delta^{\prime}$ is formed by the diagonal operators

$$
\left[\begin{array}{cccc}
\lambda_{1} I_{\mathcal{E}_{1}} & 0 & \ldots & 0 \\
0 & \lambda_{2} I_{\mathcal{E}_{2}} & \ldots & 0 \\
\vdots & \vdots & \ddots & \vdots \\
0 & 0 & \ldots & \lambda_{n} I_{\mathcal{E}_{n}}
\end{array}\right]
$$

for $\lambda_{j} \in \mathbf{C}, j=1,2, \ldots, n$, and $I_{\mathcal{E}_{j}}$ denotes the identity operator on $\mathcal{E}_{j}, j=$ $1,2, \ldots, n$. Let $A$ be any operator on $\mathcal{E}$ the $n \times n$ block matrix representation of which has entries in the set $\left\{0, A_{1}, \ldots, A_{n}\right\}$ with only one nonzero entry in each row and column. Then it is easy to check that $L\left(\Delta^{\prime} A \Delta^{\prime}\right)$ has the property required in Corollary 2, and therefore

$$
\mu_{\Delta^{\prime \prime}}(A)=\hat{\mu}_{\Delta}(A) .
$$

\section{Toeplitz Operators}

In this section, we want to use our lifting methodology in order to derive a beautiful result of Shamma $[13,14]$ on the structured singular value of a Toeplitz operator, i.e., a linear time invariant system.

Accordingly, set $\mathcal{E}=H^{2}\left(\mathbf{C}^{n}\right)$ and let $A$ denote the multiplication (analytic Toeplitz) operator on $\mathcal{E}$ defined by

$$
(A h)(z)=A(z) h(z), \quad|z|<1, h \in \mathcal{E},
$$


where

$$
A(z)=\left[a_{j k}\right]_{j, k=1}^{n}, \quad|z|<1
$$

has $H^{\infty}$ entries. Let $\Delta^{\prime}$ be any ${ }^{*}$-subalgebra of $\mathcal{L}\left(\mathbf{C}^{n}\right)$, the elements of which are regarded as multiplication operators on $\mathcal{E}$. Note that in this case, $\Delta^{\prime \prime}=\Delta$ is the algebra generated by operators of the form

$$
(B h)(z)=B(z) h(z), \quad|z|<1, h \in \mathcal{E}
$$

with $B(z) X=X B(z),|z|<1, X \in \Delta^{\prime}$ as well as of the form

$$
B\left[\begin{array}{c}
h_{1} \\
h_{2} \\
\vdots \\
h_{n}
\end{array}\right]=\left[\begin{array}{c}
Y h_{1} \\
Y h_{2} \\
\vdots \\
Y h_{n}
\end{array}\right],
$$

with $Y \in \mathcal{L}\left(H^{2}(\mathbf{C})\right)$ arbitrary. We can now state:

Lemma 11 Let $A_{0}$ be an analytic Toeplitz operator. Then if $A_{0}$ has property $(\mathcal{O})$, it also has property $\left(\mathcal{O}^{0}\right)$.

Proof. Without loss of generality we may assume $\left\|A_{0}\right\|=1$. Let $X \in \Delta^{\prime}$ and let $h_{j}, j=1,2, \ldots$ be a sequence of unit vectors satisfying

$$
\left\|\left(I-A_{0}^{*} A_{0}\right) h_{j}\right\|^{2} \rightarrow 0, \quad\left\langle\left(X-A_{0}^{*} X A_{0}\right) h_{j}, h_{j}\right\rangle \rightarrow 0 .
$$

Note that since $I-A_{0}^{*} A_{0} \geq 0$ the first condition in (9) is equivalent to

$$
\left\langle\left(I-A_{0}^{*} A_{0}\right) h_{j}, h_{j}\right\rangle \rightarrow 0 .
$$

Let $U$ denote the canonical unilateral shift on $\mathcal{E}=H^{2}\left(\mathbf{C}^{n}\right)$, that is,

$$
(U h)(z):=z h(z), \quad|z|<1, h \in \mathcal{E} \text {. }
$$

As is well-known, we can view $H^{2}\left(\mathbf{C}^{n}\right)$ as a subspace of $L^{2}\left(\mathbf{C}^{n}\right)$. In particular, in this representation the relations (9) are equivalent to

$$
\begin{aligned}
\int_{0}^{2 \pi}\left(\left\|h_{j}\left(e^{i t}\right)\right\|^{2}-\left\|A_{0}\left(e^{i t}\right) h_{j}\left(e^{i t}\right)\right\|^{2}\right) d t & \rightarrow 0 \\
\int_{0}^{2 \pi}\left[\left\langle X h_{j}\left(e^{i t}\right), h_{j}\left(e^{i t}\right)\right\rangle-\left\langle X A_{0}\left(e^{i t}\right) h_{j}\left(e^{i t}\right), h_{j}\left(e^{i t}\right)\right\rangle\right] d t & \rightarrow 0 .
\end{aligned}
$$


Note that $X$ is an $n \times n$ matrix with constant coefficients. Therefore in (10), $h_{j}$ can be replaced by $U^{k} h_{j}$ for any $k \geq 0$ without changing the values of the integrals. We infer that

$$
\left\|\left(I-A_{0}^{*} A_{0}\right) U^{k_{j}} h_{j}\right\|^{2} \rightarrow 0,\left\langle\left(X-A_{0}^{*} X A_{0}\right) U^{k_{j}} h_{j}, U^{k_{j}} h_{j}\right\rangle \rightarrow 0,
$$

for any sequence $\left\{k_{j}\right\}_{j=1}^{\infty}$ of natural numbers. Since for any $g, h \in \mathcal{E}$

$$
\left|\left\langle U^{k} g, h\right\rangle\right|=\left|\left\langle g, U^{* k} h\right\rangle\right| \leq\|g\|\left\|U^{* k} h\right\| \rightarrow 0(k \rightarrow \infty),
$$

we can choose $k_{j}$ sufficiently large in order to guarantee that

$$
\left|\left\langle U^{k_{j}} h_{j}, h\right\rangle\right| \leq \frac{1}{2^{j}},
$$

for any $h$ of the form

$$
h=\left(z^{m} \delta_{p k}\right)_{k=1}^{n}, 0 \leq m \leq j, 1 \leq p \leq n,
$$

where $\delta_{p k}$ is the Kronecker delta. Thus

$$
\left\langle U^{k_{j}} h_{j}, h\right\rangle \rightarrow 0, \text { as } j \rightarrow \infty
$$

for all vectors of the form (11). Since these vectors form an orthonormal basis of $\mathcal{E}$, we see that $U^{k_{j}} h_{j} \rightarrow 0$ weakly, which concludes the proof of the lemma. 2

Corollary $3([\mathbf{1 3}, \mathbf{1 4}])$ For $A$ and $\Delta^{\prime}$ as above, we have that

$$
\mu_{\Delta}(A)=\hat{\mu}_{\Delta}(A) .
$$

Proof. First, note that any operator $B$ in $L\left(\Delta^{\prime} A \Delta^{\prime}\right)$ is also an analytic Toeplitz operator. In particular, the critical operator $A_{0}$ obtained in the proof of Theorem 1 is a multiplication operator given by

$$
A_{0}(z)=\left[a_{j k}^{0}(z)\right]_{j, k=1}^{n},|z|<1 \text {. }
$$

By Lemma 9 , the operator $A_{0}$ has property $(\mathcal{O})$, and thus also property $\left(\mathcal{O}^{0}\right)$, by virtue of Lemma 11. The conclusion now follows from Theorem 2.2 


\section{Structured Singular Value of Input-Output Operators}

In this section, we will put some of the above results into a system-theoretic framework. Accordingly, let $\ell_{+}^{2}$ be the space of square summable one-sided sequences in $\mathrm{C}$, let $\mathcal{C}$ denote the set of all bounded linear operators on $\ell_{+}^{2}$. Further, let $A: \ell_{+}^{2}\left(\mathbf{C}^{n}\right) \rightarrow \ell_{+}^{2}\left(\mathbf{C}^{n}\right)$ be an arbitrary bounded linear operator. Thus $A$ defines a (possibly) time-varying system. (Here $\ell_{+}^{2}\left(\mathbf{C}^{n}\right)$ the space of of square summable sequences in $\mathbf{C}^{n}$, i.e., the space of finite energy vector-valued signals with $n$ components.) Then we want to interpret $\widehat{\mu}_{\Delta}(A)$ as a structured singular value on an extended space with an enhanced perturbation structure. Note $\mathcal{E}$ in this case is the Hilbert space $\ell_{+}^{2}\left(\mathrm{C}^{n}\right)$.

Define the algebra of perturbations

$$
\Delta:=\left\{\left[\begin{array}{cccc}
\delta_{1} & 0 & \ldots & 0 \\
0 & \delta_{2} & \ldots & 0 \\
\vdots & \vdots & \ddots & \vdots \\
0 & 0 & \ldots & \delta_{n}
\end{array}\right]: \delta_{i} \in \mathcal{C}, i=1, \ldots, n\right\} .
$$

Then the commutant of $\Delta$ is the finite dimensional $C^{*}$-algebra,

$$
\Delta^{\prime}:=\left\{\left[\begin{array}{cccc}
d_{1} & 0 & \ldots & 0 \\
0 & d_{2} & \ldots & 0 \\
\vdots & \vdots & \ddots & \vdots \\
0 & 0 & \ldots & d_{n}
\end{array}\right]: d_{i} \in \mathbf{C}, i=1, \ldots, n\right\} .
$$

Note that a constant $d \in \mathrm{C}$ defines an operator on $\ell_{+}^{2}$ via multiplication.

From Theorem 1, it follows that the $\mu$ upper bound given by the infimum of $\left\|X A X^{-1}\right\|$ over all constant $X$-scales equals $\bar{\mu}_{\Delta}(A)$. We now have the following interpretation of $\widehat{\mu}_{\Delta}(A)$. We lift $A$ to $A^{(n)}: \mathcal{E}^{(n)} \rightarrow \mathcal{E}^{(n)}$. Then

$$
\left(\Delta_{n}\right)^{\prime \prime} \cong\left\{\left[\begin{array}{cccc}
\tilde{\Delta}_{1} & 0 & 0 & 0 \\
0 & \tilde{\Delta}_{2} & 0 & 0 \\
\vdots & \vdots & \ddots & \vdots \\
0 & 0 & \ldots & \tilde{\Delta}_{n}
\end{array}\right]: \tilde{\Delta}_{j} \in \Delta\right\}
$$

$\left(\Delta_{n}\right)^{\prime \prime}$ is a space of time-varying perturbations and we have from Theorem 1 that

$$
\widehat{\mu}_{\Delta}(A)=\mu_{\Delta_{n}^{\prime \prime}}\left(A^{(n)}\right) .
$$


This is true for arbitrary time-varying systems $A$. In case $A$ is Toeplitz, i.e., the system is time-invariant, then as we have seen (Corollary 3, [13, 14]),

$$
\widehat{\mu}_{\Delta}(A)=\mu_{\Delta}(A) .
$$

\section{References}

[1] H. Bercovici, C. Foias, and A. Tannenbaum, "Structured interpolation theory," Operator Theory: Advances and Applications 47 (1990), pp. $195-220$.

[2] H. Bercovici, C. Foias, and A. Tannenbaum, "A spectral commutant lifting theorem," Trans. AMS $\mathbf{3 2 5}$ (1991), pp. 741-763.

[3] H. Bercovici, C. Foias, and A. Tannenbaum, "A relative ToeplitzHausdorff theorem," to appear in Operator Theory: Advances and Applications.

[4] H. Bercovici, C. Foias, and A. Tannenbaum, "Continuity of the spectrum on closed similarity orbits," Integral Equations and Operator Theory 18 (1994), 242-246.

[5] H. Bercovici, C. Foias, P. Khargonekar, and A. Tannenbaum, "On a lifting theorem for the structured singular value," to appear in Journal of Math. Analysis and Applications.

[6] J. C. Doyle, "Analysis of feedback systems with structured uncertainties," IEE Proc. 129 (1982), pp. 242-250.

[7] M. Fan, "A lifting result on structured singular values," Technical Report, Georgia Institute of Technology, Atlanta, Georgia, November 1992.

[8] P. Halmos, A Hilbert Space Problem Book, Springer-Verlag, New York, 1982.

[9] M. Khammash and J. B. Pearson, "Performance robustness of discretetime systems with structured uncertainty," IEEE Trans. Aut. Control AC-36 (1991), pp. 398-412.

[10] A. Magretski, "Power distribution approach in robust control," Technical Report, Royal Institute of Technology, Stockholm, Sweden, 1992.

[11] W. Rudin, Functional Analysis, 2nd Edition, McGraw-Hill, 1991. 
[12] M. G. Safonov, Stability Robustness of Multivariable Feedback Systems, MIT Press, Cambridge, Mass., 1980.

[13] J. Shamma, "Robust stability with time-varying structured uncertainty," to appear in IEEE Trans. Aut. Control.

[14] J. Shamma, "Robust stability for time-varying systems," 31st Proc. IEEE Conference on Decision and Control, Tucson, Arizona, 1992, pp. $3163-3168$. 